\title{
Cumulative distribution functions and moments of lattice polynomials
}

\author{
Jean-Luc Marichal \\ Applied Mathematics Unit, University of Luxembourg \\ 162A, avenue de la Fä̈encerie, L-1511 Luxembourg, Luxembourg
}

\begin{abstract}
We give the cumulative distribution functions, the expected values, and the moments of lattice polynomials when regarded as real functions. Since lattice polynomial functions include order statistics, our results encompass the corresponding formulas for order statistics.
\end{abstract}

Key words: lattice polynomial, order statistic, cumulative distribution function, moment, reliability.

\section{Introduction}

Order statistics have been deeply investigated from a probabilistic viewpoint. Their cumulative distribution functions (c.d.f.'s) as well as their expected values and moments are now well known. For theoretical developments, see e.g. the remarkable monograph by David and Nagaraja (2003).

Order statistics can be naturally extended to nonsymmetric real functions called lattice polynomial functions (Birkhoff, 1967). Roughly speaking, an $n$ ary lattice polynomial function is defined from any well-formed expression involving $n$ real variables $x_{1}, \ldots, x_{n}$ linked by the lattice operations $\wedge=\min$ and $\vee=\max$ in an arbitrary combination of parentheses. For instance,

$$
p\left(x_{1}, x_{2}, x_{3}\right)=\left(x_{1} \wedge x_{2}\right) \vee x_{3}
$$

is a 3-ary lattice polynomial function.

Email address: jean-luc.marichal [at] uni.lu (Jean-Luc Marichal). 
In this note we give closed-form formulas for the c.d.f. of any lattice polynomial function in terms of the c.d.f.'s of its input variables. More precisely, considering a lattice polynomial function $p: \mathbb{R}^{n} \rightarrow \mathbb{R}$ and $n$ independent random variables $X_{1}, \ldots, X_{n}, X_{i}(i=1, \ldots, n)$ having c.d.f. $F_{i}(x)$, we give formulas for the c.d.f. of $Y_{p}:=p\left(X_{1}, \ldots, X_{n}\right)$. We also yield formulas for the expected value $\mathbf{E}\left[g\left(Y_{p}\right)\right]$, where $g$ is any measurable function. The special cases $g(x)=x, x^{r},\left[x-\mathbf{E}\left(Y_{p}\right)\right]^{r}$, and $e^{t x}$ give, respectively, the expected value, the raw moments, the central moments, and the moment-generating function of $Y_{p}$

As the order statistics are exactly the symmetric lattice polynomial functions (Marichal, 2002), we retrieve, as a corollary, the well-known formulas of the c.d.f.'s and the moments of the order statistics.

As we will see in Section 2, any $n$-ary lattice polynomial function is completely determined by its values at the $2^{n}$ elements of $\{0,1\}^{n}$. In turn, the formulas we provide are expressed in terms of those values.

This note is organized as follows. In Section 2 we recall the basic material related to lattice polynomial functions and order statistics. In Section 3 we provide the announced results. In Section 4 we investigate the particular case where the input random variables are identically distributed. Finally, in Section 5 we provide an application of our results to the reliability analysis of coherent systems.

Lattice polynomial functions play an important role in conjoint measurement theory. Ovchinnikov (1998, Theorem 5.3) proved that the $n$-ary lattice polynomial functions are exactly those functions $f: \mathbb{R}^{n} \rightarrow \mathbb{R}$ which are continuous and order-invariant in the sense that, for any increasing bijection $\phi: \mathbb{R} \rightarrow \mathbb{R}$, we have

$$
f\left[\phi\left(x_{1}\right), \ldots, \phi\left(x_{n}\right)\right]=\phi\left[f\left(x_{1}, \ldots, x_{n}\right)\right] .
$$

Marichal (2002, Corollary 4.4) showed that, in this axiomatization, continuity can be replaced with nondecreasing monotonicity in each variable.

\section{Lattice polynomial functions}

As we shall be concerned with lattice polynomials of random variables, we will not consider lattice polynomials on a general lattice, but simply on the real line $\mathbb{R}$, which is a particular lattice. The lattice operations $\wedge$ and $\vee$ will then represent the minimum and maximum operations, respectively. To simplify the notation, we will also set $[n]:=\{1, \ldots, n\}$ for any integer $n \geqslant 1$.

Let us first recall the definition of a lattice polynomial (with real variables); 
see e.g. Birkhoff (1967, §II.5).

Definition 1 Given a finite collection of variables $x_{1}, \ldots, x_{n} \in \mathbb{R}$, a lattice polynomial in the variables $x_{1}, \ldots, x_{n}$ is defined as follows:

(1) the variables $x_{1}, \ldots, x_{n}$ are lattice polynomials in $x_{1}, \ldots, x_{n}$;

(2) if $p$ and $q$ are lattice polynomials in $x_{1}, \ldots, x_{n}$, then $p \wedge q$ and $p \vee q$ are lattice polynomials in $x_{1}, \ldots, x_{n}$;

(3) every lattice polynomial is formed by finitely many applications of the rules (1) and (2).

When two different lattice polynomials $p$ and $q$ in the variables $x_{1}, \ldots, x_{n}$ represent the same function from $\mathbb{R}^{n}$ to $\mathbb{R}$, we say that $p$ and $q$ are equivalent and we write $p=q$. For instance, $x_{1} \vee\left(x_{1} \wedge x_{2}\right)$ and $x_{1}$ are equivalent.

Because $\mathbb{R}$ is a distributive lattice, any lattice polynomial function can be written in disjunctive and conjunctive forms as follows; see e.g. Birkhoff (1967, $\S I I .5)$.

Proposition 2 Let $p: \mathbb{R}^{n} \rightarrow \mathbb{R}$ be any lattice polynomial function. Then there are nonconstant set functions $v: 2^{[n]} \rightarrow\{0,1\}$ and $w: 2^{[n]} \rightarrow\{0,1\}$, with $v(\varnothing)=0$ and $w(\varnothing)=1$, such that

$$
p(x)=\bigvee_{\substack{S \subseteq[n] \\ v(S)=1}} \bigwedge_{i \in S} x_{i}=\bigwedge_{\substack{S \subseteq[n] \\ w(S)=0}} \bigvee_{i \in S} x_{i} .
$$

The set functions $v$ and $w$ that disjunctively and conjunctively generate the polynomial function $p$ in Proposition 2 are not unique. For example, as we have already observed above, we have

$$
x_{1} \vee\left(x_{1} \wedge x_{2}\right)=x_{1}=x_{1} \wedge\left(x_{1} \vee x_{2}\right) .
$$

However, it can be shown (Marichal, 2002) that, from among all the possible set functions that disjunctively generate a given lattice polynomial function, only one is nondecreasing. Similarly, from among all the possible set functions that conjunctively generate a given lattice polynomial function, only one is nonincreasing. These particular set functions are given by

$$
v(S)=p\left(\mathbf{1}_{S}\right) \quad \text { and } \quad w(S)=p\left(\mathbf{1}_{[n] \backslash S}\right),
$$

where, for any $S \subseteq[n], \mathbf{1}_{S}$ denotes the characteristic vector of $S$ in $\{0,1\}^{n}$. Thus, an $n$-ary lattice polynomial function can always be written as

$$
p(x)=\bigvee_{\substack{S \subseteq[n] \\ p\left(\mathbf{1}_{S}\right)=1}} \bigwedge_{i \in S} x_{i}=\bigwedge_{\substack{S \subseteq[n] \\ p\left(\mathbf{1}_{[n] \backslash S}=0\right.}} \bigvee_{i \in S} x_{i}
$$


We now consider the important particular case of symmetric lattice polynomial functions. Denote by $x_{(1)}, \ldots, x_{(n)}$ the order statistics resulting from reordering the variables $x_{1}, \ldots, x_{n}$ in the nondecreasing order, i.e., $x_{(1)} \leqslant \cdots \leqslant x_{(n)}$. As Ovchinnikov $(1996, \S 7)$ observed, any order statistic is a symmetric lattice polynomial function. More precisely, for any $k \in[n]$, we have

$$
x_{(k)}=\bigvee_{\substack{S \subseteq[n] \\|S|=n-k+1}} \bigwedge_{i \in S} x_{i}=\bigwedge_{\substack{S \subseteq[n] \\|S|=k}} \bigvee_{i \in S} x_{i}
$$

Conversely, Marichal $(2002, \S 2)$ showed that any symmetric lattice polynomial function is an order statistic.

Let us denote by $o s_{k}: \mathbb{R}^{n} \rightarrow \mathbb{R}$ the $k$ th order statistic function, that is, $o s_{k}(x):=x_{(k)}$. It is then easy to see that, for any $S \subseteq[n]$, we have $\operatorname{os}_{k}\left(\mathbf{1}_{S}\right)=1$ if and only if $|S| \geqslant n-k+1$ and, likewise, we have $o s_{k}\left(\mathbf{1}_{[n] \backslash S}\right)=0$ if and only if $|S| \geqslant k$.

\section{Cumulative distribution functions and moments}

Consider $n$ independent random variables $X_{1}, \ldots, X_{n}, X_{i}(i \in[n])$ having c.d.f. $F_{i}(x)$, and set $Y_{p}:=p\left(X_{1}, \ldots, X_{n}\right)$, where $p: \mathbb{R}^{n} \rightarrow \mathbb{R}$ is any lattice polynomial function. The c.d.f. of $Y_{p}$ is given in the next theorem.

Theorem 3 Let $p: \mathbb{R}^{n} \rightarrow \mathbb{R}$ be a lattice polynomial function. Then, the c.d.f. of $Y_{p}$ is given by each of the following formulas:

$$
\begin{aligned}
& F_{p}(y)=1-\sum_{\substack{S \subseteq[n] \\
p\left(\mathbf{1}_{S}\right)=1}} \prod_{i \in[n] \backslash S} F_{i}(y) \prod_{i \in S}\left[1-F_{i}(y)\right], \\
& F_{p}(y)=\sum_{\substack{S \subseteq[n] \\
p\left(\mathbf{1}_{[n] \backslash S)=0}\right.}} \prod_{i \in S} F_{i}(y) \prod_{i \in[n] \backslash S}\left[1-F_{i}(y)\right] .
\end{aligned}
$$

Proof. Starting from the disjunctive form of $p$, we have

$$
\begin{aligned}
F_{p}(y) & =1-\operatorname{Pr}\left[\bigvee_{\substack{S \subseteq[n] \\
p\left(\mathbf{1}_{S}\right)=1}} \bigwedge_{i \in S} X_{i}>y\right] \\
& =1-\operatorname{Pr}\left[\exists S \subseteq[n], \text { with } p\left(\mathbf{1}_{S}\right)=1 \text {, such that } y<\bigwedge_{i \in S} X_{i}\right] .
\end{aligned}
$$

Consider the following events: 


$$
\begin{aligned}
& A:=\left[\exists S \subseteq[n], \text { with } p\left(\mathbf{1}_{S}\right)=1, \text { such that } y<\bigwedge_{i \in S} X_{i}\right] \\
& B:=\left[\exists S \subseteq[n], \text { with } p\left(\mathbf{1}_{S}\right)=1, \text { such that } \bigvee_{i \in[n] \backslash S} X_{i} \leqslant y<\bigwedge_{i \in S} X_{i}\right] .
\end{aligned}
$$

Event $B$ implies event $A$ trivially. Conversely, noting that $p$ is nondecreasing in each variable and replacing $S$ with a superset $S^{\prime} \supseteq S$, if necessary, we readily see that event $A$ implies event $B$.

Since the events $\left[\bigvee_{i \in[n] \backslash S} X_{i} \leqslant y<\bigwedge_{i \in S} X_{i}\right](S \subseteq[n])$ are mutually exclusive, we have

$$
F_{p}(y)=1-\sum_{\substack{S \subseteq[n] \\ p\left(\mathbf{1}_{S}\right)=1}} \operatorname{Pr}\left[\bigvee_{i \in[n] \backslash S} X_{i} \leqslant y\right] \operatorname{Pr}\left[\bigwedge_{i \in S} X_{i}>y\right]
$$

which proves the first formula. The second one can be proved similarly by starting from the conjunctive form of $p$.

The expressions of $F_{p}(y)$, given in Theorem 3, are closely related to the following concept of multilinear extension of a set function, which was introduced by Owen (1972) in game theory.

Definition 4 The multilinear extension of a set function $v: 2^{[n]} \rightarrow \mathbb{R}$ is the function $\Phi_{v}:[0,1]^{n} \rightarrow \mathbb{R}$ defined by

$$
\Phi_{v}(x):=\sum_{S \subseteq[n]} v(S) \prod_{i \in S} x_{i} \prod_{i \in[n] \backslash S}\left(1-x_{i}\right) .
$$

Using this concept, we can immediately rewrite (1) and (2) as

$$
\begin{aligned}
& F_{p}(y)=1-\Phi_{v_{p}}\left[1-F_{1}(y), \ldots, 1-F_{n}(y)\right], \\
& F_{p}(y)=\Phi_{v_{p}^{*}}\left[F_{1}(y), \ldots, F_{n}(y)\right]
\end{aligned}
$$

where $v_{p}: 2^{[n]} \rightarrow\{0,1\}$ and $v_{p}^{*}: 2^{[n]} \rightarrow\{0,1\}$ are nondecreasing set functions defined by

$$
v_{p}(S):=p\left(\mathbf{1}_{S}\right) \quad \text { and } \quad v_{p}^{*}(S):=1-p\left(\mathbf{1}_{[n] \backslash S}\right) .
$$

Owen (1972) showed that the function $\Phi_{v}$, being a multilinear polynomial, has the form

$$
\Phi_{v}(x)=\sum_{S \subseteq[n]} m_{v}(S) \prod_{i \in S} x_{i}
$$


where the set function $m_{v}: 2^{[n]} \rightarrow \mathbb{R}$, called the Möbius transform of $v$, is defined as

$$
m_{v}(S)=\sum_{T \subseteq S}(-1)^{|S|-|T|} v(T)
$$

Using this polynomial form of $\Phi_{v}$, we can immediately derive two further formulas for $F_{p}(y)$, namely

$$
\begin{aligned}
& F_{p}(y)=1-\sum_{S \subseteq[n]} m_{v_{p}}(S) \prod_{i \in S}\left[1-F_{i}(y)\right] \\
& F_{p}(y)=\sum_{S \subseteq[n]} m_{v_{p}^{*}}(S) \prod_{i \in S} F_{i}(y) .
\end{aligned}
$$

Formulas (1)-(4) thus provide four equivalent expressions for $F_{p}(y)$. As particular cases, we retrieve the c.d.f. of any order statistic. For example, using formulas (2) and (4) leads to the following corollary; see David and Nagaraja $(2003, \S 5.1)$

Corollary 5 For any $k \in[n]$, the c.d.f. of $Y_{o s_{k}}$ is given by each of the following formulas:

$$
\begin{aligned}
& F_{o s_{k}}(y)=\sum_{\substack{S \subseteq[n] \\
|S| \geqslant k}} \prod_{i \in S} F_{i}(y) \prod_{i \in[n] \backslash S}\left[1-F_{i}(y)\right], \\
& F_{o s_{k}}(y)=\sum_{\substack{S \subseteq[n] \\
|S| \geqslant k}}\left(\begin{array}{c}
|S|-1 \\
k-1
\end{array}\right)(-1)^{|S|-k} \prod_{i \in S} F_{i}(y) .
\end{aligned}
$$

Let us now consider the expected value $\mathbf{E}\left[g\left(Y_{p}\right)\right]$, where $g: \mathbb{R} \rightarrow \mathbb{R}$ is any measurable function. From its expression we can compute the expected value and the moments of $Y_{p}$.

By definition, we simply have

$$
\mathbf{E}\left[g\left(Y_{p}\right)\right]=\int_{-\infty}^{\infty} g(y) \mathrm{d} F_{p}(y)=-\int_{-\infty}^{\infty} g(y) \mathrm{d}\left[1-F_{p}(y)\right]
$$

Using integration by parts, we can derive alternative expressions of $\mathbf{E}\left[g\left(Y_{p}\right)\right]$. We then have the following immediate result.

Theorem 6 Let $p: \mathbb{R}^{n} \rightarrow \mathbb{R}$ by any lattice polynomial function and let $g$ : $\mathbb{R} \rightarrow \mathbb{R}$ be any measurable function. 
(1) If $\lim _{y \rightarrow \infty} g(y)\left[1-F_{i}(y)\right]=0(i \in[n])$, then

$$
\mathbf{E}\left[g\left(Y_{p}\right)\right]=\lim _{y \rightarrow-\infty} g(y)+\int_{-\infty}^{\infty}\left[1-F_{p}(y)\right] \mathrm{d} g(y) .
$$

(2) If $\lim _{y \rightarrow-\infty} g(y) F_{i}(y)=0(i \in[n])$, then

$$
\mathbf{E}\left[g\left(Y_{p}\right)\right]=\lim _{y \rightarrow \infty} g(y)-\int_{-\infty}^{\infty} F_{p}(y) \mathrm{d} g(y) .
$$

Clearly, combining this result with formulas (1)-(4) immediately leads to various explicit expressions of $\mathbf{E}\left[g\left(Y_{p}\right)\right]$.

\section{The case of identically distributed variables}

Let us now assume that the random variables $X_{1}, \ldots, X_{n}$ are independent and identically distributed (the i.i.d. case), each with c.d.f. $F(x)$. Clearly, this assumption makes the expressions of $\mathbf{E}\left[g\left(Y_{p}\right)\right]$ much simpler.

Example 7 Suppose $X_{1}, \ldots, X_{n}$ are uniformly distributed on $[0,1]$. In this case, for any measurable function $g:[0,1] \rightarrow \mathbb{R}$, we get

$$
\mathbf{E}\left[g\left(Y_{p}\right)\right]=g(0)+\sum_{\substack{S \subseteq[n] \\ p\left(\mathbf{1}_{S}\right)=1}} \int_{0}^{1} y^{n-|S|}(1-y)^{|S|} \mathrm{d} g(y) .
$$

We now show that, in the i.i.d. case, it is possible to express $F_{p}(y)$ in terms of $F_{\text {os }}(y)(k \in[n])$ in a very concise way.

For any lattice polynomial function $p: \mathbb{R}^{n} \rightarrow \mathbb{R}$ and any $s \in\{0, \ldots, n\}$, define the $s$-average of $p$ as

$$
\bar{p}_{s}:=\frac{1}{\left(\begin{array}{l}
n \\
s
\end{array}\right)} \sum_{\substack{S \subseteq[n] \\
|S|=s}} p\left(\mathbf{1}_{S}\right) .
$$

Theorem 8 Let $p: \mathbb{R}^{n} \rightarrow \mathbb{R}$ be a lattice polynomial function. Then, the c.d.f. of $Y_{p}$ is given by

$$
F_{p}(y)=\sum_{s=0}^{n-1} F_{o s_{n-s}}(y) \Delta_{s} \bar{p}_{s}
$$

where $\Delta_{s}$ is the difference operator, i.e., $\Delta_{s} z_{s}=z_{s+1}-z_{s}$.

Proof. Using summation by parts, we simply have 


$$
\begin{aligned}
\sum_{s=0}^{n-1} F_{o s_{n-s}}(y) \Delta_{s} \bar{p}_{s} & =\left.\bar{p}_{s} F_{o s_{n-s}}(y)\right|_{0} ^{n}-\sum_{s=0}^{n-1} \bar{p}_{s+1} \Delta_{s} F_{o s_{n-s}}(y) \\
& =1-\sum_{s=1}^{n} \bar{p}_{s} \Delta_{s} F_{o s_{n-s+1}}(y) .
\end{aligned}
$$

However, by Corollary 5, we have

$$
\Delta_{s} F_{o s_{n-s+1}}(y)=\left(\begin{array}{l}
n \\
s
\end{array}\right) F(y)^{n-s}[1-F(y)]^{s} \quad(s=0, \ldots, n) .
$$

Therefore,

$$
\sum_{s=0}^{n-1} F_{o s_{n-s}}(y) \Delta_{s} \bar{p}_{s}=1-\sum_{\substack{S \subseteq[n] \\ p\left(\mathbf{1}_{S}\right)=1}} F(y)^{n-|S|}[1-F(y)]^{|S|}=F_{p}(y)
$$

Recall that, in the i.i.d. case, $F_{o s_{k}}(y)$ can be written as (see David and Nagaraja, 2003, §2.1)

$$
F_{o s_{k}}(y)=I_{F(y)}(k, n-k+1),
$$

where $I_{z}(a, b)$ is the regularized Beta function defined, for any $a, b, z>0$, by

$$
I_{z}(a, b):=\frac{\int_{0}^{z} t^{a-1}(1-t)^{b-1} \mathrm{~d} t}{\int_{0}^{1} t^{a-1}(1-t)^{b-1} \mathrm{~d} t} .
$$

It follows immediately from Theorem 8 that

$$
F_{p}(y)=\sum_{s=0}^{n-1} I_{F(y)}(n-s, s+1) \Delta_{s} \bar{p}_{s}
$$

\section{Application to reliability theory}

In this final section we show how the results derived here can be applied to the reliability analysis of certain coherent systems. For a reference on reliability theory, see e.g. Barlow and Proschan (1981).

Consider a system made up of $n$ independent components, each component $C_{i}(i \in[n])$ having a lifetime $X_{i}$ and a reliability $r_{i}(t):=\operatorname{Pr}\left[X_{i}>t\right]$ at time $t>0$.

We assume that, when components are connected in series, the lifetime of the subsystem they form is simply given by the minimum of the component 
lifetimes. Likewise, for a parallel connection, the subsystem lifetime is the maximum of the component lifetimes.

It follows immediately that, for a system mixing series and parallel connections, the system lifetime is given by a lattice polynomial function

$$
Y_{p}=p\left(X_{1}, \ldots, X_{n}\right)
$$

of the component lifetimes. We then have explicit formulas for the c.d.f., the expected value, and the moments of the system lifetime.

For example, the system reliability at time $t>0$ is given by

$$
R_{p}(t):=\operatorname{Pr}\left[Y_{p}>t\right]=\Phi_{v_{p}}\left[r_{1}(t), \ldots, r_{n}(t)\right] .
$$

Moreover, for any measurable function $g:[0, \infty[\rightarrow \mathbb{R}$ such that

$$
\lim _{t \rightarrow \infty} g(t) r_{i}(t)=0 \quad(i \in[n])
$$

we have, by Theorem 6 ,

$$
\mathbf{E}\left[g\left(Y_{p}\right)\right]=g(0)+\int_{0}^{\infty} R_{p}(t) \mathrm{d} g(t) .
$$

Example 9 If $r_{i}(t)=e^{-\lambda_{i} t}(i \in[n])$, we can easily show that

$$
\mathbf{E}\left[Y_{p}^{k}\right]=\sum_{\substack{S \subseteq[n] \\ S \neq \varnothing}} m_{v_{p}}(S) \frac{k !}{\lambda(S)^{k}} \quad(k \in \mathbb{N}),
$$

where $\lambda(S):=\sum_{i \in S} \lambda_{i}$. In particular, for a series system we retrieve the wellknown formula $\mathbf{E}\left[Y_{p}^{k}\right]=\frac{k !}{\lambda([n])^{k}}$. For a parallel system we have

$$
\mathbf{E}\left[Y_{p}^{k}\right]=\sum_{\substack{S \subseteq[n] \\ S \neq \varnothing}}(-1)^{|S|-1} \frac{k !}{\lambda(S)^{k}} .
$$

\section{Acknowledgments}

The author is indebted to N. Balakrishnan and A. Dukhovny for their comments during the preparation of this paper. He also wants to thank the reviewer who pointed out the applicability of the results to the reliability theory. 


\section{References}

Barlow, R.E., Proschan, F., 1981. Statistical theory of reliability and life testing. To Begin With, Silver Spring, MD.

Birkhoff, G., 1967. Lattice theory. Third edition. American Mathematical Society Colloquium Publications, Vol. XXV. American Mathematical Society, Providence, R.I.

David, H., Nagaraja, H., 2003. Order statistics. 3rd ed. Wiley Series in Probability and Statistics. Chichester: John Wiley \& Sons.

Marichal, J.-L., 2002. On order invariant synthesizing functions. J. Math. Psych. 46 (6), 661-676.

Ovchinnikov, S., 1996. Means on ordered sets. Math. Social Sci. 32 (1), 39-56.

Ovchinnikov, S., 1998. Invariant functions on simple orders. Order 14 (4), $365-371$.

Owen, G., 1972. Multilinear extensions of games. Management Science 18, P64-P79. 